\title{
Photolittérature, M. Caraion et J.-P. Montier (dir.)
}

\section{Francesca Forcolin}

\section{(2) OpenEdition}

\section{Journals}

\section{Edizione digitale}

URL: http://journals.openedition.org/studifrancesi/10638

DOI: 10.4000/studifrancesi. 10638

ISSN: 2427-5856

\section{Editore}

Rosenberg \& Sellier

\section{Edizione cartacea}

Data di pubblicazione: 1 décembre 2017

Paginazione: 604-605

ISSN: 0039-2944

\section{Notizia bibliografica digitale}

Francesca Forcolin, «Photolittérature, M. Caraion et J.-P. Montier (dir.)», Studi Francesi [Online], 183 (LXI I III) | 2017, online dal 01 février 2018, consultato il 21 janvier 2021. URL: http://

journals.openedition.org/studifrancesi/10638; DOI: https://doi.org/10.4000/studifrancesi.10638

Questo documento è stato generato automaticamente il 21 janvier 2021.

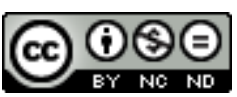

Studi Francesi è distribuita con Licenza Creative Commons Attribuzione - Non commerciale - Non opere derivate 4.0 Internazionale. 


\title{
Photolittérature, M. Caraion et J.-P. Montier (dir.)
}

\author{
Francesca Forcolin
}

\section{NOTIZIA}

Photolittérature, sous la direction de Marta CARAION et Jean-Pierre MONTIER, Fondation Jan Michalski pour l'écriture et la littérature, Montricher, 2017, 177 pp.

1 Per introdurre la natura del volume, possono essere citate le parole di Véronique Montémont, autrice dell'articolo presente nella collettanea La boite à histoires (pp. 134-135): «Même si les images sont "illustratives", convoquées comme on place un décor en toile de fond, leur présence rompt le flux de la lecture, qui doit céder la place, pour quelques minutes ou quelques secondes, à la contemplation». Come in una sorta di mise en abîme, il volume propone 12 contributi incentrati sul rapporto tra letteratura $\mathrm{e}$ fotografia, intervallati da numerose rappresentazioni di pagine tratte da opere letterarie che presentano al loro interno fotografie. Dopo uno o più contributi, dunque, il lettore cede per qualche minuto alla contemplazione di estratti di poesie, romanzi e saggi tra le cui righe s'insinuano corpi (Man Ray), paesaggi (Doisneau, Kertész), scene di vita parigina (Brassai), ritratti di famiglia (Simon).

2 Photolittérature è un catalogo nato dalla mostra omonima che ha avuto luogo dal 14 ottobre al 20 dicembre 2016 a Montricher, sostenuta e organizzata dalla Fondazione svizzera Jan Michalski e curata da M. CARAION et J.-P. MONTIER che firmano l'Introduction (pp. 6-7). L'esposizione intende rendere conto della complessa relazione tra parole e immagini che si snoda nel corso di quasi due secoli-testimoni delle ibridazioni e dell'evoluzione dei supporti editoriali - a partire dalla nascita della settima arte, percorrendo correnti estetiche e generi differenti (racconti di viaggio, autobiografia, poesia). Lo scopo è ragionare sulle varie forme di interazione tra le due arti: dialoghi tra scrittori e fotografi; fotografi che diventano scrittori e scrittori-fotografi; testi illustrati con fotografie anonime; testi in cui l'immagine, assente, è il tema di fondo. Documento 
assoluto perché in grado di catturare un tempo e uno spazio precisi, utopia di una memoria totale, la fotografia inizialmente è stata causa di dissapori (testimone il severo giudizio di Baudelaire in occasione del Salon de peinture del 1859), per poi aprire la strada a dialoghi e interazioni feconde a partire dall'esperienza avanguardistica del Surrealismo. In seguito, con la seconda metà del xx secolo la vertigine della scrittura in prima persona trova nella fotografia un interessante documento testimoniale, attribuendole la missione della restituzione della memoria collettiva e individuale.

Gli articoli sono suddivisi in sei sezioni. La prima, «L'apparition de la photographie: entre rejet et fascination», si apre con Jean-Pierre MONTIER, Extension du domaine du photolittéraire (pp. 10-12), analisi generale sull'apporto della fotografia dai suoi esordi: essa, introducendosi nel terreno della mimesis, è rivoluzione invisibile insidiando il campo della letteratura; è strumento della modernità; è causa di una rivoluzione epocale e fenomeno in continua espansione, estendendosi in un campo molto vasto di fenomeni sociali e artistici. Segue Paul EDWARDS con Grandes étapes dans l'élaboration matérielle du photobook de fiction littéraire en langue française (pp. 14-18), intervento a carattere storico, incentrato sulla nascita ed evoluzione dei procedimenti tecnici - dalla calotipia alla quadricromia fino all'offset odierno-che vedono la definizione e il perfezionamento dei volumi illustrati. Le pagine successive riportano estratti di Champfleury, Villiers de l'Isle-Adam, opere di Daudet con illustrazioni di Magron, o, nel caso di Les frères Kip, il testo di Verne è accompagnato da immagini di un autore ignoto. Segue la seconda parte, "Voyages et portraits des pays», con il contributo di Marta CARAION, Le voyage, des origines de la photolittérature au XXe siècle (pp. 36-39), la quale sottolinea l'innegabile e proficua collaborazione tra scrittori e fotografi nell'ambito della letteratura di viaggio, già esplicitata da Baudelaire che concede alla fotografia il solo merito di arricchire "l'album du voyageur". Quest'arte, infatti, offre al racconto di viaggio una garanzia di reale iscrivendosi anche in un progetto testimoniale: basti pensare agli scatti di Du Camp in Oriente e, in epoca più attuale, alle Lettres d'Egypte di Berger e Guibert. David MARTENS, in Portraits de territoires (pp. 40-41), si sofferma sui ritratti paesaggistici dagli anni Venti in poi, vedendo uno sviluppo decisivo a cavallo tra le guerre, periodo in cui la ville lumière attira molteplici sguardi. Ne sono un esempio le pagine di Révoil con immagini del Karkar, Bruges-la Morte di Rodenbach, le fotografie di una Parigi decadente di Brassai o di Kertész, la banlieue ripresa da Doisneau, la campagna secondo Strand. Si passa alle avanguardie con la sezione «Chassés-croisés à l'ère des avant-gardes", che ospita il solo articolo di Andrea OBERHUBER, Une ceuvre, deux signatures: la part du photographique dans le livre surréaliste (pp. 68-70), riflessione sul solido benché sconosciuto contributo dei fotografi ai testi surrealisti, che appaiono ancora meno conosciuti dei pittori o dei disegnatori: si pensi a Nadja, raramente ricordata per le immagini di Boiffard. Le opere a quattro mani tra scrittori e fotografi risultano un vero ideale per la pratica surrealista, inaugurata con Les champs magnetiques, esempio per eccellenza di androginia creatrice che rompe con l'immagine romantica del soggetto creatore solitario. Seguono frammenti tratti da Kodak di Cendrars, Le Printemps di Mac Orlan, volti femminili in estasi catturati da Dalì, corpi nudi da Man Ray, pagine da L'amour fou di Breton. La quarta parte, «Le roman-photo», presenta un altro contributo di Marta CARAION, Le roman photo au tournant du siècle ou la liberté de collaborer (pp. 84-85): i ritratti posti sui biglietti da visita, scattati in studi fotografici dall'ambientazione borghese stereotipata, vanno ad impregnare la produzione letteraria di fine Ottocento che presenta intrighi borghesi, il mondo dello 
spettacolo e un Oriente erotizzato. Jan BAETENS, in Les récits du roman-photo (pp. 86-88), si sofferma sul fotoromanzo, genere nato nel 1947 che appartiene principalmente alla letteratura sentimentale, e che in seguito ha trovato sviluppo anche nelle serie televisive: genere parodiato ma che ha notevolmente influito su diversi linguaggi. Seguono estratti da opere di Loti, come Les trois dames de la Kasbah; La dame turque di Lorrain con fotografie di autori ignoti; opere di Gyp con immagini della borghesia parigina; racconti di Simenon arricchiti da fotografie di Krull. La quinta parte, «Poétiques et récits photolittéraires», si apre con Jean-Pierre MONTIER, La question de la fiction: frictions productives entre image et graphie (pp. 110-115), che si concentra sul rapporto tra fotografia e reale: se essa, come affermava Barthes, è troppo vicina al reale da non poter scavallare nell'immaginario, la sua inserzione nella logica letteraria tradizionale è allora inammissibile se non scandalosa. Il suo utilizzo nella finzione riguarda pertanto i campi più strettamente della conoscenza, del sapere, come traccia di documento e archivio. All'articolo seguono estratti da opere di Simon che firma il testo e le fotografie familiari di Album d'un amateur; da Le Feu di Novarina con fotografie di Joly; il dialogo tra le due arti tra De Kerangal e Grimbert. Véronique MONÉMONT nel sopracitato intervento La boîte à histoires studia l'interesse contemporaneo per la scrittura in prima persona corredata da fotografie di vita familiare (Garat, Ernaux); oppure opere che non considerano l'immagine come elemento di accompagnamento, ma come punto di partenza per una riflessione sul sé e sulla natura del testo (Calle, Narbo). Seguono fotografie di Boltanski, Guibert, Roche, dove il testo ha la sola funzione di descrivere l'immagine; pagine di Calle tratte da Douleur exquise e di Desplechin da L'Album vert. Philippe ORTEL, in Littérature et photographie au XIX siècle: la référence invisible (pp. 150-152), definisce la fotografia come "spectrale", in quanto solo negli ultimi decenni ha ottenuto riconoscimento da parte delle istituzioni, e soltanto la letteratura le ha permesso di accedere alla scena sociale. Antonin WISER, in Un autoportrait au sujet manquant (pp. 154-155), si rifà invece a quei testi dove l'immagine è protagonista pur non essendoci: la fotografia è evocata, conservata dall'autore e mantenuta implicita; oppure invocata perché sparita, distrutta, dimenticata; simulata perché inesistente; allucinata in quanto immagine impossibile e fantasmata, "assoluta" come in Duras. Seguono estratti da Simon, Guibert, Garat, Modiano, Ernaux. Chiude il lavoro la sesta parte, «L'espace métalittéraire», che contiene il solo articolo di Servanne MoNjouR, Les nouvelles inventions littéraires de la photographie (pp. 172-175), riflessione sull'evoluzione contemporanea dell'immagine: gli scrittori si appropriano fecondamente dei nuovi dispositivi che investono anche la struttura letteraria, accantonando, anche solo momentaneamente, il cartaceo a profitto del digitale. 